\title{
CCL28 promotes breast cancer growth and metastasis through MAPK-mediated cellular anti-apoptosis and pro-metastasis
}

\author{
XIAO LI YANG ${ }^{1,2^{*}}$, KAI YI LIU ${ }^{3 *}$, FENG JUAN LIN ${ }^{1,2}$, HUI MIN SHI ${ }^{2}$ and ZHOU LUO OU ${ }^{1,2}$ \\ ${ }^{1}$ Breast Cancer Institute, Key Laboratory of Breast Cancer in Shanghai, Fudan University Shanghai Cancer Center, \\ Shanghai 200032; ${ }^{2}$ Department of Oncology, Shanghai Medical College, Fudan University, Shanghai 200032; \\ ${ }^{3}$ Department of Biology, College of Life and Environmental Sciences, \\ Shanghai Normal University, Shanghai 200234, P.R. China
}

Received November 5, 2016; Accepted May 29, 2017

DOI: $10.3892 /$ or.2017.5798

\begin{abstract}
Breast cancer is one of the most commonly diagnosed cancers worldwide and the second leading cause of cancerrelated deaths among females. CCL28 (mucosa-associated epithelial chemokine, MEC), a CC subfamily chemokine, has been well studied in the process of inflammation, and recently increasing evidence indicates that CCL28 is related to tumor progression. However, little is known concerning its function in breast cancer. In the present study, we generated a CCL28overexpressing breast cancer cell line MDA-MB-231HM/ CCL28 from parental MDA-MB-231HM cells. We found that overexpression of CCL28 promoted cell proliferation and tumor formation, and also enhanced migration, invasion and metastasis both in vitro and in vivo. Mechanistic studies revealed that CCL28 mediated intracellular activation of the mitogen-activated protein kinase (MAPK) signaling pathway to promote breast cancer cell proliferation and metastasis by upregulating anti-apoptotic protein $\mathrm{Bcl}-2$ and suppressing cell adhesion protein $\beta$-catenin. However, overexpression of CCL28 did not influence the expression of metastasis-related protein matrix metalloproteinase MMP2 and MMP9 and VEGF. Tissue sample analysis from animal models also indicated that overexpression of CCL28 was associated with enhanced pERK expression and reduced $\beta$-catenin expression in breast carcinomas. Thus, our results show for the first time that CCL28 contributes to breast cancer progression through the ERK/MAPK-mediated anti-apoptotic and metastatic signaling pathway. Antagonists of CCL28 and the MAPK signaling pathway may be used synergistically to treat breast cancer patients.
\end{abstract}

Correspondence to: Dr Zhou Luo Ou, Breast Cancer Institute, Key Laboratory of Breast Cancer in Shanghai, Fudan University Shanghai Cancer Center, Shanghai 200032, P.R. China

E-mail: ouzhouluo@163.com

*Contributed equally

Key words: CCL28, MAPK, breast cancer, metastasis, proliferation

\section{Introduction}

Breast cancer is one of the most common malignancies among females worldwide. As a result of early detection and improved treatment, the mortality of breast cancer patients has been declining. However, metastasis is still the leading cause of death among breast cancer patients, accounting for more than $90 \%$ of cancer-related mortality $(1,2)$. Although many studies have focused on the mechanisms of carcinogenesis and metastasis in breast cancer, the exact mechanism remains unclear.

Chemokines and their receptors have been widely known to play important roles in multiple pathways of tumor progression, including cancer cell proliferation, survival, cellular senescence, angiogenesis and metastasis (3). Among the four subfamilies (CXC, CC, CX3C and XC) of chemokines, the subfamily of $\mathrm{CC}$ chemokines has attracted more and more attention in recent years. For example, CCL18 was reported to promote breast cancer metastasis (4) and induce epithelialto-mesenchymal transition in lung cancer (5). CCL2 (MCP-1) was shown to facilitate breast cancer metastasis (6), promote cancer stem cell (CSC)-mediated disease progression (7) and enhance breast cancer cell survival (8).

CCL28, also known as mucosa-associated epithelial chemokine (MEC), which is a ligand for CCR3/CCR10, was initially recognized as a chemokine predominantly produced by epithelial cells in diverse mucosal tissues (9), including the salivary gland, mammary gland, trachea, colon, and to a lesser extent in the small intestine (10). Moreover, its expression can be increased after stimulation with pro-inflammatory cytokines and bacterial products $(11,12)$. CCL28 appears to have dual functions in different types of human cancer. For example, Dimberg et al (13) found that CCL28 protein expression in colon tumors was significantly lower than that in normal tissues, suggesting that suppression of CCL28 expression was related to colorectal carcinogenesis. Moreover, CCL28 mRNA and protein expression were markedly reduced in pleomorphic adenomas and adenolymphomas compared with the levels in normal adjacent tissue (14). On the contrary, several studies have indicated that overexpression of CCL28 promoted tumor tolerance and angiogenesis (15). Although CCL28 was found to be produced in the mammary gland, the role of CCL28 in breast carcinogenesis and metastasis has not been explored. 
In a previous study, CCL28 mRNA expression was highly reduced or eliminated in human breast tumors compared to that noted in normal adjacent tissues, but the relevance needs to be further studied (16). In this study, we used a stably expressing CCL28 breast cancer cell line MDA-MB-231HM/ CCL28 derived from parental MDA-MB-231HM cells to investigate the effects of CCL28 on breast cancer cell proliferation, migration and invasion in vitro and in vivo. We found for the first time that CCL28 promotes breast cancer growth and metastasis at least in part through mitogen-activated protein kinase (MAPK) signal-mediated anti-apoptosis and promoted invasiveness.

\section{Materials and methods}

Cell lines and culture. Human breast cancer cell line MDA-MB-231 was obtained from the American Type Culture Collection (ATCC; Manassas, VA, USA). The MDA-MB-231HM cell line was established by a subclone selection procedure derived from MDA-MB-231 cells in our institute. The MDA-MB-231HM cell line has a high potential to metastasize to the lung and its establishment has been described previously (17). Cells were routinely maintained in the recommended Dulbecco's modified Eagle's medium (DMEM) supplemented with $10 \%$ fetal bovine serum (FBS), $100 \mathrm{U} / \mathrm{ml}$ penicillin and $100 \mu \mathrm{g} / \mathrm{ml}$ streptomycin. The cultures were incubated at $37^{\circ} \mathrm{C}$ in a humidified $5 \% \mathrm{CO}_{2}$ atmosphere. Cell culture medium and FBS were purchased from Life Technologies, Inc. (Rockville, MD, USA). RT-PCR reagents were obtained from Takara Biotechnology Co., Ltd.

TRIzol reagent (Invitrogen, Carlsbad, CA, USA) was used to extract total RNA according to the manufacturer's recommendations. CCL28 cDNA was produced by reverse transcription-polymerase chain reaction. First, reverse transcription was performed with random hexamer primers by using the PrimeScript ${ }^{\mathrm{TM}}$ RT kit (Takara Biotechnology Co., Ltd.). The primers used to amplify CCL28 cDNA were 5'-TAT GGATCCATGCAGCAGAGAGGACTCGCCAT-3' (sense, boldface type indicates the Bam HI site) and 5'-ACGCGTCGAC CTAATAAGGAGTTTTATGGCCGTA-3' (antisense, boldface type indicates the $\mathrm{SalI}$ site). Conditions for the polymerase chain reaction were $94^{\circ} \mathrm{C}$ for $2 \mathrm{~min}, 94^{\circ} \mathrm{C}$ for $30 \mathrm{sec}, 56^{\circ} \mathrm{C}$ for $1 \mathrm{~min}$, and $72^{\circ} \mathrm{C}$ for $1 \mathrm{~min}$ for a total of 35 cycles, followed by $72^{\circ} \mathrm{C}$ for a $10-$ min extension. The CCL28 reverse transcriptionpolymerase chain reaction product was purified on a $1 \%$ agarose gel, digested with BamHI and SalI, and ligated to a $\mathrm{pBabe/puromycin} \mathrm{retroviral} \mathrm{vector} \mathrm{that} \mathrm{had} \mathrm{been} \mathrm{digested} \mathrm{with}$ the same enzymes. After transformation of the plasmid in Escherichia coli (DH5 $\alpha$ ), positive clones were selected and DNA sequencing analysis was performed at the DNA sequencing corporation. The control vector used in this study was an empty pBabe/puromycin retroviral vector. All of these plasmids were transfected into amphotropic Phoenix packaging cells to generate retroviruses, which were used to infect the corresponding cell lines by using previously described protocols (18). Retroviruses carrying CCL28 cDNA were used to infect the MDA-MB-231HM cells.

RNA extraction and real-time quantitative PCR. TRIzol reagent (Invitrogen) was used to extract total RNA according to the manufacturer's recommendations. In brief, $1.0 \mu \mathrm{g}$ of total RNA was applied for reverse transcription in $20 \mu \mathrm{l}$. The quantification of mRNA levels was carried out using DNA Engine Opticon 2 real-time PCR detection system (MJ Research) with SYBR-Green. Two microliters of diluted cDNA was used as a template; $10 \mu 12 X$ SYBR Premix Ex Taq (Takara Biotechnology Co., Ltd.) was mixed with the template and primers. The total reaction volume was $20 \mu \mathrm{l}$. Cycling conditions were denaturation at $95^{\circ} \mathrm{C}$ for $30 \mathrm{sec}$, annealing at $60^{\circ} \mathrm{C}$ for $30 \mathrm{sec}$, and elongation at $72^{\circ} \mathrm{C}$ for $45 \mathrm{sec}$. Plate reading was at 72 and $82^{\circ} \mathrm{C}$. The specific primers used in the experiment were as follows: CCL28 forward, 5'-TGCACGGAG GTTTCACATCAT-3' and reverse, 5'-TTGGCAGCTTGC ACTTTCATC-3'; and GAPDH forward, 5'-ACCCACTCC TCCACCTTTGA-3' and reverse, 5'-CATACCAGGAAATG AGCTTGACAA-3'. All experiments were repeated in triplicate. To ensure that the correct product was amplified in the reaction, all PCR products were also separated using $1.2 \%$ agarose gel electrophoresis.

Western blot analysis. Total protein extract for each cell line was obtained by using a lysis buffer as described elsewhere (19), and equal amounts (30 $\mu \mathrm{g} / \mathrm{load})$ were analyzed by immuno-blotting. The antibody against $\beta$-actin was obtained from Sigma-Aldrich (A5441, 1:20,000). Antibodies against Bak (sc-832, 1:1,000), Bcl-2 (sc-7382, 1:500), matrix metalloproteinase (MMP)-9 (sc-6840, 1:1,000), MMP-2 (sc-13594, 1:1,000), vascular endothelial growth factor (VEGF, sc-507, 1:1,000) and JNK (sc-571, 1:1,000) were from Santa Cruz Biotechnology. Antibodies against ERK1/2 (9102, 1:1,000), phospho-ERK1/2 (4376, 1:1,000), MEK1/2 (9126, 1:1,000), phospho-MEK1/2 (2338, 1:1,000) and phospho-JNK (4668, 1:1,000) were obtained from Cell Signaling Technology. The antibody against Bcl-XL (\#AM05, 1:1,000) was from Calbiochem. The antibody against CCL28 (18214-1-AP, 1:500) was from Proteintech. The antibody against E-cadherin (BD 610182, 1:1,000) was obtained from BD Labware (Franklin Lakes, NJ, USA). The secondary antibodies were $\mathrm{F}(\mathrm{ab})_{2}$ fragment of donkey anti-mouse immunoglobulin (product NA931) or of donkey anti-rabbit immunoglobulin (product NA9340) linked to horseradish peroxidase from Amersham Biosciences (Little Chalfont, Buckghamshire, UK).

Proliferation assay. Cell proliferation was detected using Cell Counting Kit-8 (CCK-8; Dojindo, Kumamoto, Japan). The cells were plated in 96-well plates at a density of 2,500/well $(100 \mu \mathrm{l})$ and cultured in growth medium. The number of cells was counted according to the protocol of the kit from the company.

Wound healing assay. To evaluate cell motility, a wound healing assay was performed. The cells were cultured in a 6-well culture plate. Twenty four hours later, when the cells reached $90 \%$ confluence, a single wound was created in the center of the cell monolayer by gently scratching the attached cells with a sterile $10-\mu 1$ micropipette tip. The debris was removed by washing at least twice with phosphate-buffered saline (PBS). After 12 or $20 \mathrm{~h}$ of incubation, the cells which migrated into the wounded area or protruded from the border of the wound were visualized and photographed under an 
A

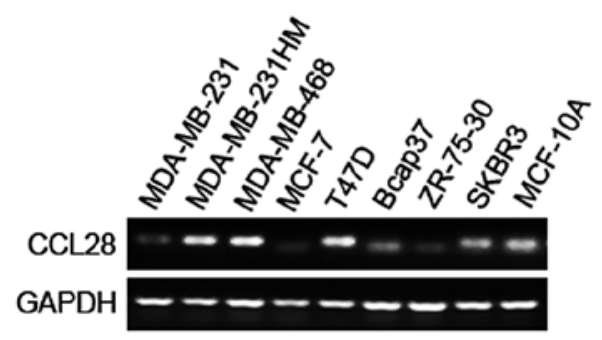

B

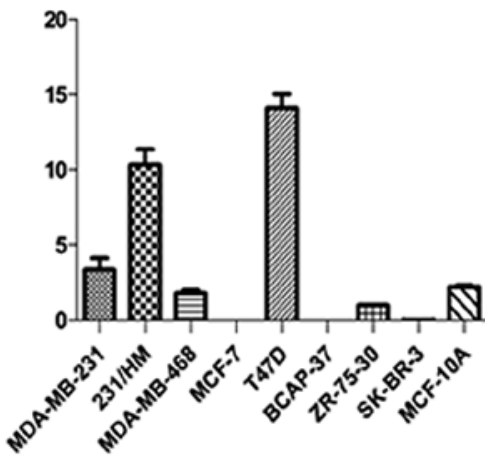

Figure 1. Expression of CCL28 in breast cancer cell lines. Expression of CCL28 mRNA was assessed by semi-quantitative RT-PCR (A) and real-time PCR (B) in 1 normal breast epithelial cell line (MCF-10A) and 8 breast cancer cell lines.

inverted microscope. Each experiment was performed at least three times independently.

In vitro invasion. Invasion experiments were carried out with a Matrigel invasion chamber (BD Labware). Each well insert was layered with $120 \mathrm{ml}$ of a 1:3 mixture of Matrigel/DMEM $\left(1,400 \mathrm{mg}\right.$ Matrigel $\left./ \mathrm{cm}^{2}\right)$. An amount of $10^{5}$ cells were incubated in an incubator at $37^{\circ} \mathrm{C}$ for $24 \mathrm{~h}$. Invasion was assessed by counting the cells that had traveled across the filter and that had attached to the bottom side of the filter. Then, the filter was fixed in $4 \%$ formalin and stained with $1 \%$ Crystal violet. Cells that had invaded through the Matrigel and reached the lower surface of the filter were counted under a light microscope at a magnification of x200. Five fields were counted for each sample.

Anchorage-independent colony formation. The soft agar assay was carried out as described previously (19). Briefly, $2 \times 10^{4}$ cells were suspended in $2 \mathrm{ml}$ of medium with $0.7 \%$ agarose LMP (A8350; Solarbio, Amresco), and the suspension was placed on top of $3 \mathrm{ml}$ of solidified $1.4 \%$ agarose. Triplicate cultures of each well type were maintained for 14 days at $37^{\circ} \mathrm{C}$ in an atmosphere of $5 \% \mathrm{CO}_{2}$ and $95 \%$ air, with fresh medium being added at day 7 . The number of colonies that were larger than $50 \mu \mathrm{m}$ ( $\sim 100$ cells) in diameter in each dish was counted at 14-20 days. The assay was repeated three times with duplicate samples.

Xenograft tumors in nude mice. All mouse experiments were carried out in accordance with the NIH 'Guide for the Care and Use of Laboratory Animals'. The study protocol was approved by the Shanghai Medical Experimental Animal Care Committee. All applicable international, national, and institutional guidelines for the care and use of animals were followed. Breast tumor cells were implanted into the mammary fat pad as described previously (20). Briefly, we harvested $3 \times 10^{6}$ cells by incubation in trypsin-EDTA, washed the cells twice with PBS and resuspended the cells in $0.15 \mathrm{ml}$ of PBS. The tumor growth of the modified and control cell lines was monitored until the day that the mice were sacrificed. The date on which the first grossly visible tumor appeared for subcutaneous injection was recorded, and the tumor size was measured every 3 days. Two-dimensional measurements were taken with an electronic caliper after injection, and tumor volume was calculated with the use of the following formula: tumor volume (in $\mathrm{mm}^{3}$ ) $=\mathrm{a} \mathrm{x} \mathrm{b}^{2} \times 0.52$, where $\mathrm{a}$ is the longest diameter, $b$ is the shortest diameter, and 0.52 is a constant to calculate the volume of an ellipsoid. Mice were sacrificed when observed for lethargy, poor appetite and feebleness. Metastasis formation was assessed by macroscopic observation of all major organs for secondary tumors and confirmed by histological examination of the organs. All tumor nodules were counted and dissected; each primary tumor nodule was also weighed and its volume was determined as described above for subcutaneous tumors.

Immunohistochemical staining for animal xenografts. Sections from the xenografts of the MDA-MB-231HM/ CCL28 and MDA-MB-231HM/vector groups were used for CCL28 and related protein detection. Antibodies used for immunohistochemical staining included anti-CCL28, anti-phospho-ERK1/2 and anti- $\beta$-catenin. Antibody binding was detected using avidinbiotin-peroxidase methods. Briefly, tissue slides were deparaffinized in xylene and rehydrated in a graded series of ethanol, and sections were subjected to antigen retrieval by boiling in $0.01 \mathrm{~mol} / 1$ sodium citrate buffer $(\mathrm{pH} 6.0)$ in a microwave oven for $10 \mathrm{~min}$. After blocking endogenous peroxidase activity with $0.3 \%$ hydrogen peroxide and blocking nonspecific protein binding with $1.5 \%$ normal goat serum, the sections were incubated overnight with an antibody at $4{ }^{\circ} \mathrm{C}$ in a humid chamber. Then, antibodies were localized by incubating sections with biotinylated goat anti-mouse or goat anti-rabbit IgG for $30 \mathrm{~min}$ and detected with 3,3-diaminobenzidine (DAB). The lung and lymph tissues were serially cut into 5- $\mu \mathrm{m}$ slices, and every 10th section was stained with hematoxylin and eosin (H\&E) to evaluate the presence or the absence of lung metastasis. Two independent pathologists calculated the number of metastasis in whole lungs.

Statistical analysis. Statistical analysis was performed using Statistical Package for the Social Sciences (SPSS) software version 16.0 for Windows (SPSS Inc., Chicago, IL, USA). ANOVA and Student's t-test were used to determine the statistical significance of differences between experimental groups 

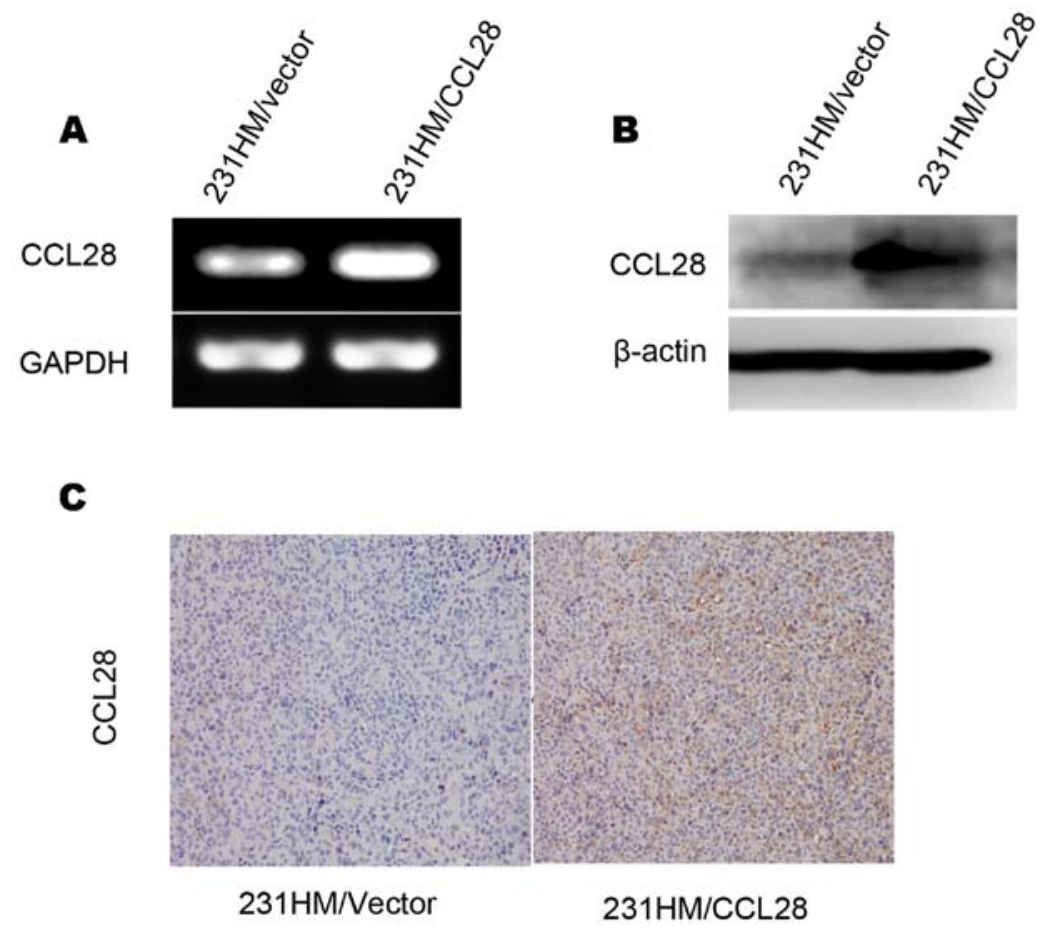

Figure 2. Stable transfection of CCL28 cDNA into MDA-MB-231HM cells. (A) Expression of CCL28 mRNA was detected by RT-PCR in MDA-MB-231HM cells that were transfected with control (231HM/vector) or CCL28 cDNA (231HM/CCL28). Expression of CCL28 protein was detected by western blot analysis in 231HM/vector and 231HM/CCL28 cell lines (B) and immunohistochemistry analysis in xenografts of 231HM/vector and 231HM/CCL28 tumor bearing mice $(\mathrm{C})$.

in vitro. Values of $\mathrm{p}<0.05$ were considered as statistically significant. Graphs were created with GraphPad Prism 5.

\section{Results}

Expression of CCL28 in human breast cancer cell lines. CCL28 mRNA expression was detected by reverse transcription-PCR in 8 breast cancer cell lines (MCF-7, MDA-MB-468, ZR-75-30, MDA-MB-231, MDA-MB-231HM, SKBR3, Bcap37, T47D) and a normal mammary epithelial cell line (MCF-10A) (Fig. 1A). To further confirm the difference in CCL28 mRNA expression in these cells, we detected CCL28 mRNA expression by quantitative RT-PCR (Fig. 1B). We found that CCL28 mRNA expression was high in the MDA-MB-231HM and T47D cell lines, moderate in the MDA-MB-231, MDA-MB-468, ZR-75-30 and MCF-10A cell lines, and low in the other cell lines. It was clear that CCL28 mRNA expression was higher in high-metastatic breast cancer cell line MDA-MB-231HM than that in low-metastatic cell line MDA-MB-231 and in MCF-7 a nonmetastatic cell line, which indicated a potential correlation between the expression of CCL28 and the metastatic ability of breast cancer cells.

Stable transfection of CCL28 CDNA into MDA-MB-231HM cells. In order to investigate the role of CCL28 in breast cancer cell tumorigenesis and metastasis, we transfected CCL28 expression vector $\mathrm{pBabe/CCL28}$ into MDA-MB-231HM cells and generated stable transfectants. As shown by reverse transcription-PCR (Fig. 2A), western blot analysis (Fig. 2B) and immunohistochemistry (Fig. 2C) in the tissues generated from animals injected with MDA-MB-231HM/CCL28 or MDA-MB-231HM/vector, CCL28 was markedly over-expressed in the cells respectively treated with CCL28 cDNA (MDA-MB-231HM/CCL28) compared with the control cells treated with the empty vector (MDA-MB-231HM/vector). These results suggest that a stable expressing CCL28 breast cancer cell line was successfully generated, and it can be used in further experiments.

CCL28 promotes breast cancer cell proliferation, tumorigenesis and tumor growth. To investigate whether expression of CCL28 is associated with tumor growth of breast cancer cells in vitro, we performed proliferation and anchorageindependent growth assays in MDA-MB-231HM/CCL28 cells and compared the results with the parental cells. As shown in Fig. 3A, overexpression of CCL28 significantly promoted breast cancer cell proliferation. Furthermore, the number of colonies formed in soft agar was increased in the MDA-MB-231HM/CCL28 cells compared to that noted in the control cells (Fig. 3B). Furthermore, western blot analysis showed that the expression of anti-apoptotic protein Bcl-2 was higher in the MDA-MB-231HM/CCL28 cells when compared with that in the control cells, whereas no changes were observed in the expression of Bcl-XL. The pro-apoptotic protein BAK was decreased in the MDA-MB-231HM/CCL28 cells (Fig. 3C).

Next, the effect of CCL28 on tumor growth was further investigated using an orthotropic xenograft tumor model in nude mice. The results showed that MDA-MB-231HM/CCL28 cell-derived tumors grew much faster than the MDA-MB-231HM/vector cell-derived tumors in the nude mice (Fig. 3D). When the mice were xenografted with MDA-MB-231HM/CCL28 cells, as shown in Fig. 3E, the 
A

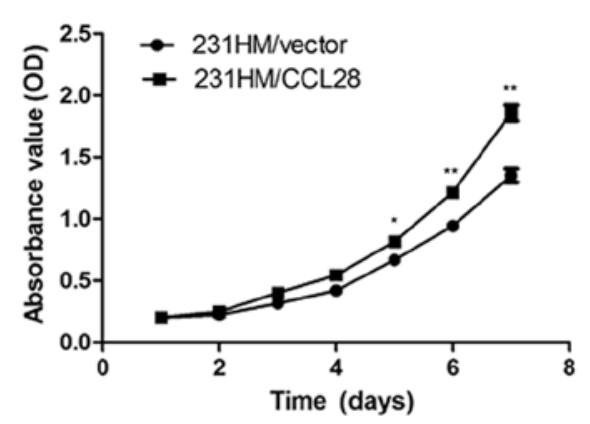

D

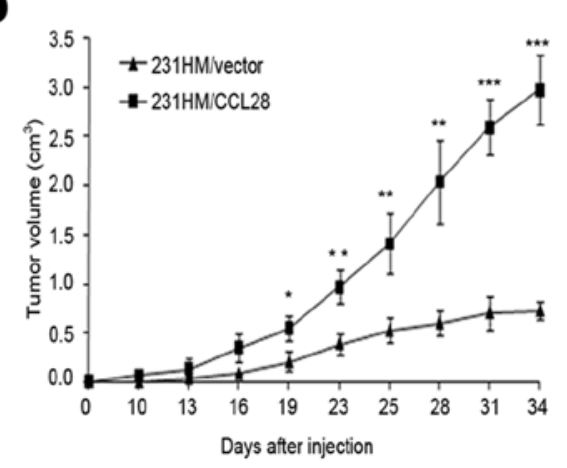

B

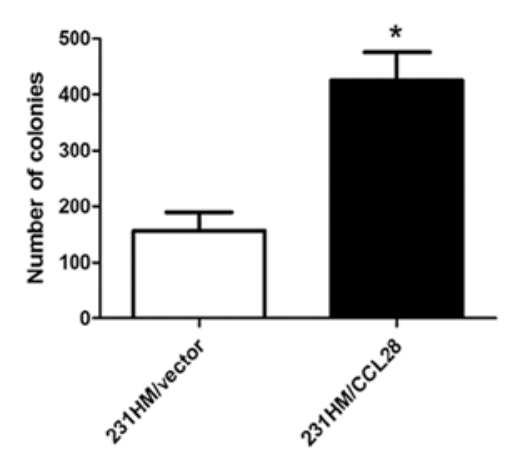

E

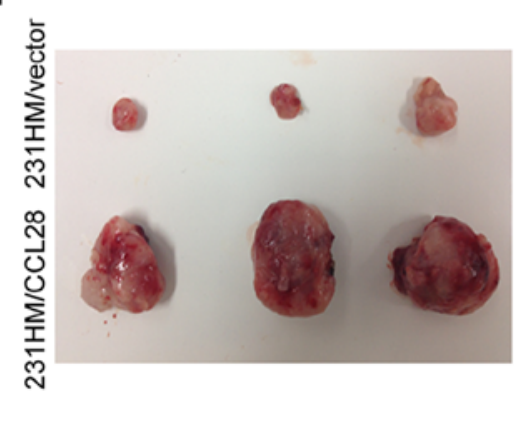

C

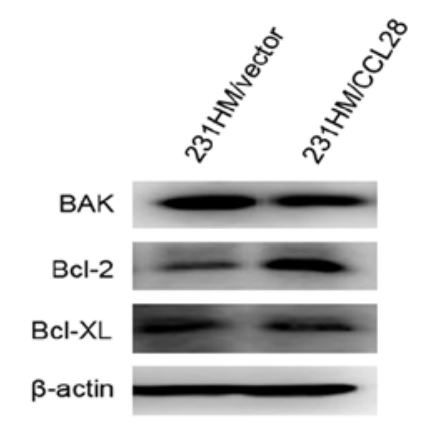

$\mathbf{F}$

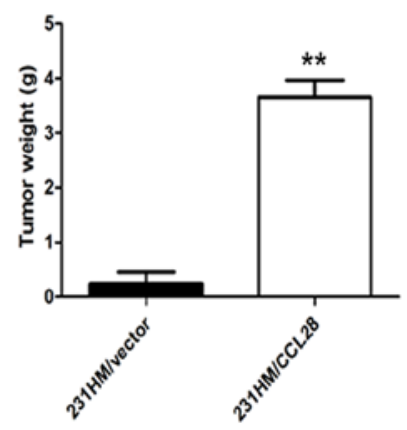

Figure 3. CCL28 promotes breast cancer cell proliferation, tumorigenesis and tumor growth. (A) Growth curve of MDA-MB-231HM/vector (231HM/vector) and MDA-MB-231HM/CCL28 (231HM/CCL28) cells as determined by a proliferation assay. Values shown are means \pm standard deviation (SD). ${ }^{\text {p }}<0.05$, ${ }^{* *} \mathrm{P}<0.01$. Data are from three independent experiments with triplicate samples. (B) Anchorage independent colony formation on soft agar of $231 \mathrm{HM} / \mathrm{vector}$ and 231HM/CCL28 cells. Error bars, 95\% CIs. Data from three independent experiments are shown. * $<0.05$. (C) Western blot analysis of apoptosis-related protein expression. $\beta$-actin was used as a loading control. (D) Tumor growth curve of $231 \mathrm{HM} /$ vector and $231 \mathrm{HM} / \mathrm{CCL} 28$ cell xenografts in vivo. ${ }^{*}$ p $<0.05$, ${ }^{* *}$ p $<0.01$, ${ }^{* * *} \mathrm{p}<0.001$. (E) Gross volume of CCL28-overexpressing tumors and vector control tumors. (F) The average tumor weight of $231 \mathrm{HM} / \mathrm{CCL} 28$ xenografts and control xenografts. ${ }^{* *} \mathrm{p}<0.01$.

primary tumor volume was much larger than that noted for the control cells. Furthermore, the average tumor weight was also increased in the mice bearing MDA-MB-231HM/CCL28 xenografts as compared with the control xenografts (Fig. 3F). Collectively, these data suggest that CCL28 enhances breast cancer cell proliferation, tumorigenesis and tumor growth.

CCL28 enhances breast cancer cell migration, invasion and tumor metastasis. We then assessed the invasiveness of MDA-MB-231HM/CCL28 and parental cells using Transwell chambers. The results showed that more MDA-MB-231HM/CCL28 cells intruded into the bottom chamber than the number of MDA-MB-231HM/vector cells (Fig. 4A). A wound-healing assay was used to evaluate the effect of CCL28 expression on breast cancer cell migration. As shown in Fig. 4B, MDA-MB-231HM/CCL28 cells migrated more rapidly close to the scratched wound when compared to the MDA-MB-231HM/vector cells within $20 \mathrm{~h}$. Thus, overexpression of CCL28 increased cell migration and invasion in vitro.

We also investigated the effect of CCL28 on the metastasis of tumors in vivo. As shown in Fig. 4C, H\&E staining showed that CCL28 led to more massive metastasis in the lungs and lymph node of the mice injected with MDA-MB-231HM/CCL28 cells than the metastasis of mice injected with the control cells. Injection of MDA-MB-231HM/CCL28 cells also increased the number of metastatic nodules in the lungs and lymph nodes (Fig. 4C).
MAPK pathway is involved in CCL28-mediated tumor metastasis. To investigate the potential mechanism of CCL28 in breast cancer metastasis, we first examined the expression of metastasis-associated proteins in cells overexpressing CCL28, and found that no significant changes could be detected in the levels of MMP2, MMP9 and VEGF, while the expression of $\beta$-catenin was decreased (Fig. 5A). $\beta$-catenin is a dual function protein, involved in the regulation and coordination of cell-cell adhesion and gene transcription. The induced expression is related to breast cancer (21). In this study, further immunohistochemistry analysis showed that $\beta$-catenin expression was decreased in the xenograft mouse tumor tissues overexpressing CCL28, which was consistent with the western blot results (Fig. 5B). These data suggest that CCL28 promotes breast cancer cell metastasis through these molecules.

Since the MAPK pathway is associated with the promotion of tumor metastasis, we examined the expression of signal molecules involved in the MAPK pathway in the CCL28 cDNA-treated cells as well as in their corresponding control cells.As showninFig.5C,there were nochanges in the expression of ERK1/2, MEK1/2 and JNK1/2, however, levels of phosphorylated ERK1/2 (Thr202/Tyr204), MEK1/2 (Ser212/221) and JNK1/2 (Thr183/Tyr185) were markedly increased after overexpression of CCL28 compared with the control cell line. To confirm this observation, we then examined pERK expression in the tissues generated from animals injected with the MDA-MB-231HM/CCL28 and MDA-MB-231HM/vector by 
A
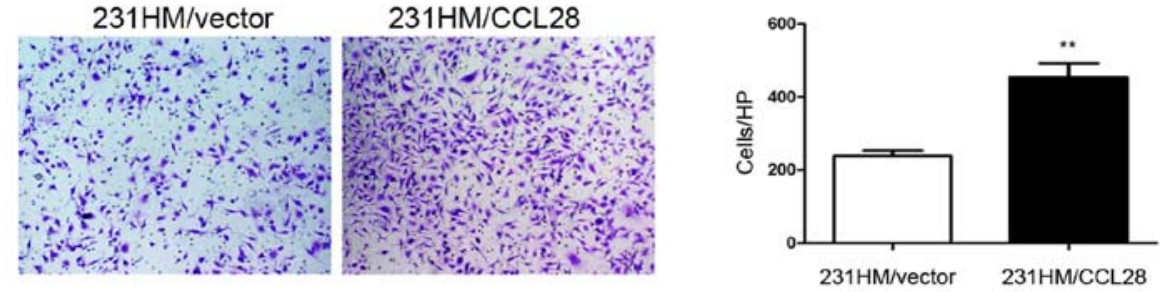

B
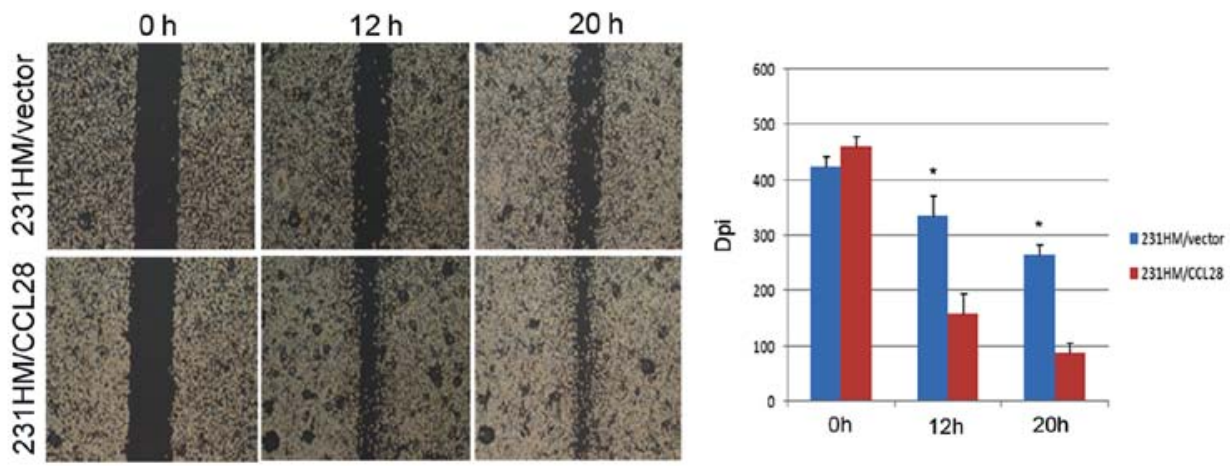

C
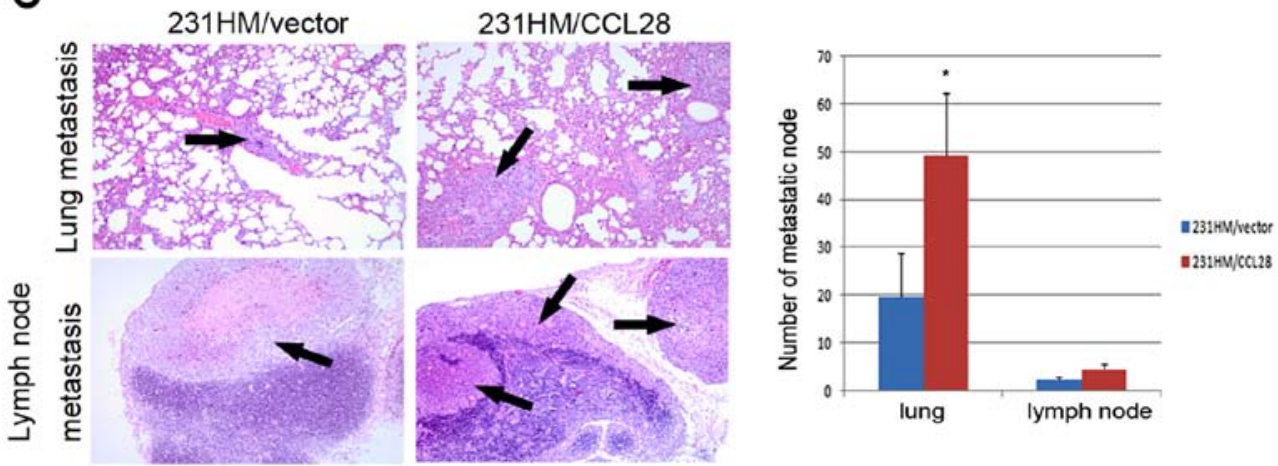

Figure 4. CCL28 enhances breast cancer cell migration, invasion and tumor metastasis. (A) Transwell assay of MDA-MB-231HM/vector (231HM/vector) and MDA-MB-231HM/CCL28 (231HM/CCL28) cells plated on the upper cell culture inserts, with culture medium alone plated in the lower chambers Quantification of the Transwell assay (right). Cells were counted in triplicate wells and in three identical experiments. ${ }^{* *} \mathrm{p}<0.01$. Columns indicate the mean of three independent experiments; bars SD; HP, high-power objective. (B) Cell migration of 231HM/CCL28 cells in a wound-healing assay. Cell migration was assayed at a magnification of $x 10$. Quantitative analysis of the wound-healing assay (right). Data from three independent experiments were used in this analysis. Values shown are means \pm standard deviation (SD). " $\mathrm{p}<0.05$ compared with the untreated group. (C) H\&E staining of pulmonary and lymph node metastatic tumors (left). Quantification of the average metastasis of each lung or lymph node of the control xenografts and CCL28-modified xenografts (right). "p $<0.05$. $\mathrm{H} \& \mathrm{E}$, hematoxylin and eosin staining.

immunohistochemistry. As expected, the expression of pERK was significantly increased (Fig. 5D). These results suggest that CCL28 promotes breast cancer metastasis by suppressing $\beta$-catenin, which may be mediated through upstream signaling of MAPK.

\section{Discussion}

Chemokines are a family of small chemotactic cytokines, which have been divided into four main subfamilies: CXC, $\mathrm{CC}, \mathrm{CX} 3 \mathrm{C}$ and $\mathrm{XC}$. Chemokines and their receptors have been widely investigated in human cancers, including breast cancer. In addition, they play a dual role - positive and negative - in cancer progression. For example, atypical chemokine receptors DARC (22), D6 (23) and CCX-CKR (24) have been found to inhibit breast cancer growth and metastasis and are positively correlated with enhanced survival, whereas CXCR2 has been demonstrated to promote ovarian cancer growth through multiple pathways (18). CCL28 is one of the newly discovered CC subfamily chemokine. Previous studies have shown that CCL28 is constitutively and inductively expressed in diverse mucosal sites, and is suggested to take part in mucosal immunity (25). Moreover, it also has a broad spectrum of antimicrobial activity $(26,27)$.

The balance of proliferation and apoptosis plays a significant role in the control of tumor growth. In general, progression of tumor growth is characterized by a net increase in the number of tumor cells. This could be due to increased proliferation and/or decreased apoptosis, or both (28). Bcl-2 and BCL-xL facilitate G0 quiescence by decreasing RNA content and cell size and upregulating p27 protein (29). In mammary tumor and hepatocellular carcinogenesis models, Bcl-2 plays 


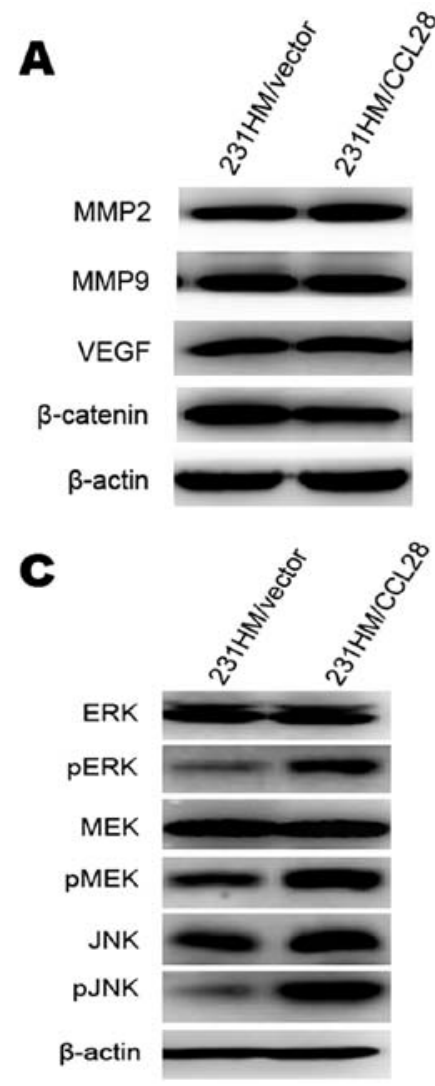

B

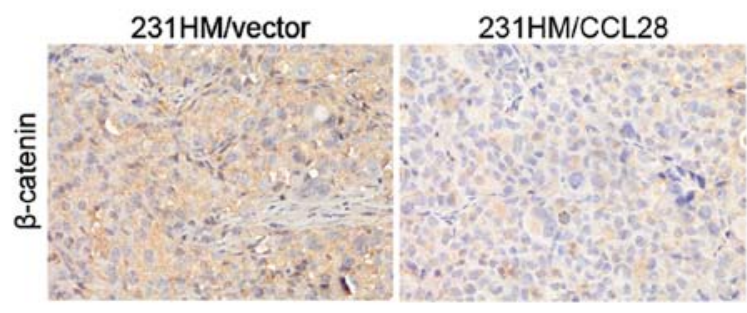

D

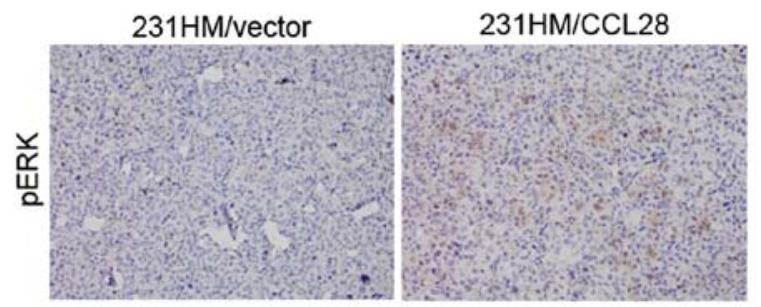

Figure 5. The MAPK pathway is involved in CCL28-mediated tumor metastasis. (A) Western blot analysis of MMP2, MMP9, VEGF and $\beta$-catenin expression in MDA-MB-231HM/vector (231HM/vector) and MDA-MB-231HM/CCL28 (231HM/CCL28) cells. (B) $\beta$-catenin protein expression was detected by immunohistochemical analysis in xenografts of 231HM/vector and 231HM/CCL28 tumor-bearing mice. (C) Association of CCL28 with the MAPK signaling pathway. Overexpression of CCL28 increased the level of phosphorylated ERK1/2, MEK1/2, and JNK1/2 as determined by western blot analysis (D) Immunohistochemical examination of pERK protein expression in xenografts of 231HM/vector and 231HM/CCL28 tumor-bearing mice. MAPK, mitogen-activated protein kinase; MMP, matrix metalloproteinase; VEGF, vascular endothelial growth factor.

a tumor-suppressing role by inhibiting proliferation (30). The MAPK pathway is highly conserved among eukaryotes and transmits signals from cell surface receptors for nuclear transcription. The MAPK family consists of ERK1/2, JNK and p38. Generally, upregulation and activation of ERK1/2 and JNK tends to promote tumor development $(31,32)$. In this study, we demonstrated that CCL28 plays an important role in breast cancer progression by promoting breast cancer cell proliferation, tumorigenesis, migration and invasion in vitro, and enhancing tumor growth and metastasis in vivo via the MAPK signaling pathway. We showed that overexpression of CCL28 increased the expression of anti-apoptotic Bcl-2, and suppressed the expression of pro-apoptotic Bak, leading to decreased cellular apoptosis. Furthermore, CCL28 promoted cell invasiveness and tumor metastasis by suppressing the expression of cell adhesion protein $\beta$-catenin.

As a CC subfamily chemokine, CCL28 and its receptors CCR3/CCR10 have been extensively investigated in terms of eosinophil recruitment in inflammation-induced diseases $(25,33)$. Hanamoto et al found that H-RS cells in 15 of 19 cases were positive for CCL28. Among them, 7 cases were also positive for CCR10, suggesting a potential autocrine effect (34). CCL28 is a key regulator of IgA ASC accumulation in the mammary gland and thus controls the passive transfer of IgA antibodies from mother to infant (35), but there is no report about whether it has an effect on cancer cells in an autocrine or a paracrine manner. There is much evidence to show that CCR3 and CCR10, two receptors for CCL28, play important roles in numerous types of malignancies (36-38), whereas the function of their ligand in epithelial cancer has not been well defined. A study in asthma demonstrated that phosphorylation of $\mathrm{NF}-\kappa \mathrm{B}$ is associated with the expression of inducible CCL28 (39). Kagami et al (40) demonstrated that CCL28 production was downregulated in keratinocytes by inhibition of ERK/MAPK and NF- $\kappa \mathrm{B}$ pathway. We showed here that CCL28 may promote breast tumorigenesis by regulating the MAPK signaling pathway, which ultimately controls cell apoptosis and metastasis.

To our knowledge, no investigation has focused on the effect of CCL28 on breast cancer cell proliferation and invasive abilities in vitro and in vivo. In our study, we showed for the first time that overexpression of CCL28 led to increased cell proliferation and tumor growth, and the effect was at least in part attributed to increased anti-apoptotic factor Bcl-2. This is consistent with a previous study in human hematopoietic stem and progenitor cells (HSPCs), which showed that CCL28 is a growth factor that directly stimulates the proliferation of primitive hematopoietic cells (41). However, Sun et al (42) obtained the opposite results and found that CCL28 induced the apoptosis of decidual stromal cells in human spontaneous abortion. The reason for the different result may be due to the different source of CCL28, different microenvironment, and different 
types of diseases, which need to be further studied in the future. Furthermore, our data showed that overexpression of CCL28 not only promoted the growth of tumors, but also elevated the metastatic ability by increasing the number of lung metastatic nodules and the weight of wet lung. Immunohistochemistry indicated that CCL28 positively regulated cancer metastasis by suppressing the expression of $\beta$-catenin. The result was confirmed by western blot analysis as following the overexpression of CCL28, the expression of $\beta$-catenin was decreased. In addition, our data showed that VEGF protein expression did not change in terms of CCL28 expression, which was not consistent with a study by Facciabene et al. They demonstrated that CCL28 production was upregulated by hypoxia, and then recruited Treg cells expressing CCR10, and ultimately promoted tumor immune tolerance and angiogenesis (15). This discrepancy may suggest that CCL28 regulates tumor progression through different mechanisms.

In summary, we demonstrated for the first time that overexpression of CCL28 promotes breast cancer proliferation and tumor growth by activating Bcl-2 expression, and increases breast cancer invasiveness and metastatic ability by suppressing $\beta$-catenin expression, which are positively related to activation of the MAPK signaling pathway. Thus, targeting CCL28 may effectively inhibit breast cancer cell growth and metastasis and may be a valuable therapeutic strategy against breast cancer.

\section{Acknowledgements}

The present study was funded by the National Natural Science Foundation of China (grant no. 81172506), and the Shanghai Committee of Science and Technology, China (grant no. 12DZ2260100).

\section{References}

1. Chaffer CL and Weinberg RA: A perspective on cancer cell metastasis. Science 331: 1559-1564, 2011.

2. Siegel R, Naishadham D and Jemal A: Cancer statistics, 2012. CA Cancer J Clin 62: 10-29, 2012.

3. Mantovani A, Savino B, Locati M, Zammataro L, Allavena P and Bonecchi R: The chemokine system in cancer biology and therapy. Cytokine Growth Factor Rev 21: 27-39, 2010

4. Chen J, Yao Y, Gong C, Yu F, Su S, Chen J, Liu B, Deng H, Wang F, Lin L, et al: CCL18 from tumor-associated macrophages promotes breast cancer metastasis via PITPNM3. Cancer Cell 19: 541-555, 2011.

5. Ploenes T, Scholtes B, Krohn A, Burger M, Passlick B, Müller-Quernheim J and Zissel G: CC-chemokine ligand 18 induces epithelial to mesenchymal transition in lung cancer A549 cells and elevates the invasive potential. PLoS One 8: e53068, 2013.

6. Qian BZ, Li J, Zhang H, Kitamura T, Zhang J, Campion LR, Kaiser EA, Snyder LA and Pollard JW: CCL2 recruits inflammatory monocytes to facilitate breast-tumour metastasis. Nature 475: 222-225, 2011.

7. Tsuyada A, Chow A, Wu J, Somlo G, Chu P, Loera S, Luu T, Li AX, Wu X, Ye W, et al: CCL2 mediates cross-talk between cancer cells and stromal fibroblasts that regulates breast cancer stem cells. Cancer Res 72: 2768-2779, 2012.

8. Fang WB, Jokar I, Zou A, Lambert D, Dendukuri P and Cheng N: CCL2/CCR2 chemokine signaling coordinates survival and motility of breast cancer cells through Smad3 protein- and p42/44 mitogen-activated protein kinase (MAPK)-dependent mechanisms. J Biol Chem 287: 36593-36608, 2012.

9. Wang W, Soto H, Oldham ER, Buchanan ME, Homey B, Catron D, Jenkins N, Copeland NG, Gilbert DJ, Nguyen N, et al: Identification of a novel chemokine (CCL28), which binds CCR10 (GPR2). J Biol Chem 275: 22313-22323, 2000.
10. Pan J, Kunkel EJ, Gosslar U, Lazarus N, Langdon P, Broadwell K, Vierra MA, Genovese MC, Butcher EC and Soler D: A novel chemokine ligand for CCR10 and CCR3 expressed by epithelial cells in mucosal tissues. J Immunol 165: 2943-2949, 2000.

11. Shibata S, Maeda S, Maeda S, Chimura N, Kondo N and Fukata T: Augmentation of CCL17 and CCL28 gene expression by TNF-alpha, IL-1beta, or IFN-gamma in cultured canine keratinocytes. Res Vet Sci 88: 422-426, 2010.

12. Ogawa H, Iimura M, Eckmann L and Kagnoff MF: Regulated production of the chemokine CCL28 in human colon epithelium. Am J Physiol Gastrointest Liver Physiol 287: G1062-G1069, 2004.

13. Dimberg J, Hugander A and Wågsäter D: Protein expression of the chemokine, CCL28, in human colorectal cancer. Int J Oncol 28: 315-319, 2006.

14. Liu GX, Lan J, Sun Y, Hu YJ and Jiang GS: Expression of the chemokine CCL28 in pleomorphic adenoma and adenolymphoma of the human salivary glands. Exp Ther Med 4: 65-69, 2012.

15. Facciabene A, Peng X, Hagemann IS, Balint K, Barchetti A, Wang LP, Gimotty PA, Gilks CB, Lal P, Zhang L, et al: Tumour hypoxia promotes tolerance and angiogenesis via CCL28 and T(reg) cells. Nature 475: 226-230, 2011.

16. Mickanin CS, Bhatia U and Labow M: Identification of a novel $\beta$-chemokine, MEC, down-regulated in primary breast tumors. Int J Oncol 18: 939-944, 2001.

17. Li JY, Ou ZL, Yu SJ, Gu XL, Yang C, Chen AX, Di GH, Shen ZZ and Shao ZM: The chemokine receptor CCR4 promotes tumor growth and lung metastasis in breast cancer. Breast Cancer Res Treat 131: 837-848, 2012.

18. Yang G, Rosen DG, Liu G, Yang F, Guo X, Xiao X, Xue F, Mercado-Uribe I, Huang J, Lin SH, et al: CXCR2 promotes ovarian cancer growth through dysregulated cell cycle, diminished apoptosis, and enhanced angiogenesis. Clin Cancer Res 16: 3875-3886, 2010.

19. Yang G, Rosen DG, Mercado-Uribe I, Colacino JA, Mills GB, Bast RC Jr, Zhou C and Liu J: Knockdown of p53 combined with expression of the catalytic subunit of telomerase is sufficient to immortalize primary human ovarian surface epithelial cells. Carcinogenesis 28: 174-182, 2007.

20. Jones LW, Viglianti BL, Tashjian JA, Kothadia SM, Keir ST, Freedland SJ, Potter MQ, Moon EJ, Schroeder T, Herndon JE II, et al: Effect of aerobic exercise on tumor physiology in an animal model of human breast cancer. J Appl Physiol (1985) 108: 343-348, 2010.

21. Aaltomaa S, Kärjä V, Lipponen P, Isotalo T, Kankkunen JP, Talja M and Mokka R: Reduced alpha- and beta-catenin expression predicts shortened survival in local prostate cancer. Anticancer Res 25: 4707-4712, 2005.

22. Wang J, Ou ZL, Hou YF, Luo JM, Shen ZZ, Ding J and Shao ZM: Enhanced expression of Duffy antigen receptor for chemokines by breast cancer cells attenuates growth and metastasis potential. Oncogene 25: 7201-7211, 2006.

23. Wu FY, Ou ZL, Feng LY, Luo JM, Wang LP, Shen ZZ and Shao ZM: Chemokine decoy receptor d6 plays a negative role in human breast cancer. Mol Cancer Res 6: 1276-1288, 2008.

24. Feng LY, Ou ZL, Wu FY, Shen ZZ and Shao ZM: Involvement of a novel chemokine decoy receptor CCX-CKR in breast cancer growth, metastasis and patient survival. Clin Cancer Res 15: 2962-2970, 2009.

25. Eksteen B, Miles A, Curbishley SM, Tselepis C, Grant AJ, Walker LS and Adams DH: Epithelial inflammation is associated with CCL28 production and the recruitment of regulatory T cells expressing CCR10. J Immunol 177: 593-603, 2006.

26. Hieshima K, Ohtani H, Shibano M, Izawa D, Nakayama T, Kawasaki Y, Shiba F, Shiota M, Katou F, Saito T, et al: CCL28 has dual roles in mucosal immunity as a chemokine with broad-spectrum antimicrobial activity. J Immunol 170: 1452-1461, 2003.

27. Jiang P, Lan J, Hu Y, Li D and Jiang G: Enhancing CCL28 expression through the gene transfer to salivary glands for controlling cariogenic microbe. Cytokine 59: 94-99, 2012.

28. Mattern $\mathbf{J}$ and Volm M: Imbalance of cell proliferation and apoptosis during progression of lung carcinomas. Anticancer Res 24: 4243-4246, 2004.

29. Kordezangeneh M, Irani S, Mirfakhraie R, Esfandyari-Manesh M, Atyabi F and Dinarvand R: Regulation of BAX/BCL2 gene expression in breast cancer cells by docetaxel-loaded human serum albumin nanoparticles. Med Oncol 32: 208, 2015.

30. Eom YH, Kim HS, Lee A, Song BJ and Chae BJ: BCL2 as a subtype-specific prognostic marker for breast cancer. J Breast Cancer 19: 252-260, 2016. 
31. Chen J, Hou R, Zhang X, Ye Y, Wang Y and Tian J: Calycosin suppresses breast cancer cell growth via ER $\beta$-dependent regulation of IGF-1R, p38 MAPK and PI3K/Akt pathways. PLoS One 9: e91245, 2014.

32. Yang XL, Lin FJ, Guo YJ, Shao ZM and Ou ZL: Gemcitabine resistance in breast cancer cells regulated by PI3K/AKT-mediated cellular proliferation exerts negative feedback via the MEK/MAPK and mTOR pathways. Onco Targets Ther 7 : 1033-1042, 2014.

33. Takeda A, Baffi JZ, Kleinman ME, Cho WG, Nozaki M, Yamada K, Kaneko H, Albuquerque RJ, Dridi S, Saito K, et al: CCR3 is a target for age-related macular degeneration diagnosis and therapy. Nature 460: 225-230, 2009.

34. Hanamoto H, Nakayama T, Miyazato H, Takegawa S, Hieshima K, Tatsumi Y, Kanamaru A and Yoshie O: Expression of CCL28 by Reed-Sternberg cells defines a major subtype of classical Hodgkin's disease with frequent infiltration of eosinophils and/or plasma cells. Am J Pathol 164: 997-1006, 2004.

35. Wilson E and Butcher EC: CCL28 controls immunoglobulin (Ig)A plasma cell accumulation in the lactating mammary gland and IgA antibody transfer to the neonate. J Exp Med 200: 805-809, 2004.

36. Miyagaki T, Sugaya M, Murakami T, Asano Y, Tada Y, Kadono T, Okochi H, Tamaki K and Sato S: CCL11-CCR3 interactions promote survival of anaplastic large cell lymphoma cells via ERK1/2 activation. Cancer Res 71: 2056-2065, 2011.
37. Kakinuma T and Hwang ST: Chemokines, chemokine receptors, and cancer metastasis. J Leukoc Biol 79: 639-651, 2006.

38. Murakami T, Cardones AR, Finkelstein SE, Restifo NP, Klaunberg BA, Nestle FO, Castillo SS, Dennis PA and Hwang ST: Immune evasion by murine melanoma mediated through $\mathrm{CC}$ chemokine receptor-10. J Exp Med 198: 1337-1347, 2003.

39. O'Gorman MT, Jatoi NA, Lane SJ and Mahon BP: IL-1beta and TNF-alpha induce increased expression of CCL28 by airway epithelial cells via an NFkappaB-dependent pathway. Cell Immunol 238: 87-96, 2005.

40. Kagami S, Saeki H, Komine M, Kakinuma T, Nakamura K, Tsunemi Y, Sasaki K, Asahina A and Tamaki K: CCL28 production in $\mathrm{HaCaT}$ cells was mediated by different signal pathways from CCL27. Exp Dermatol 15: 95-100, 2006.

41. Karlsson C, Baudet A, Miharada N, Soneji S, Gupta R, Magnusson M, Enver T, Karlsson G and Larsson J: Identification of the chemokine CCL28 as a growth and survival factor for human hematopoietic stem and progenitor cells. Blood 121: 3838-3842, 2013

42. Sun C, Zhang YY, Tang CL, Wang SC, Piao HL, Tao Y, Zhu R, Du MR and Li DJ: Chemokine CCL28 induces apoptosis of decidual stromal cells via binding CCR3/CCR10 in human spontaneous abortion. Mol Hum Reprod 19: 676-686, 2013. 